\title{
VACUUM ARC DISCHARGE ON INTEGRALLY COLD CATHODE
}

\author{
A.A. Lisenkov ${ }^{1}$, V.P. Valuev ${ }^{2}$, V.T. Barchenko ${ }^{3}$, O.I. Grebnev ${ }^{3}$, \\ N.V. Krupovich ${ }^{3}$
}

1 Institute of problems of mechanical engineering of Russian Academy of Science, Russia, SaintPetersburg

2 Saint-Petersburg State Engineering Institute (LMZ-VTUZ), Russia, Saint-Petersburg

3 St.-Petersburg State Electrotechnical University "LETI", Russia, Saint-Petersburg

\begin{abstract}
This article describes the vacuum arc plasma source of extended design generating the directed belt plasma stream and operating in pulse mode. The researches carried out give additional information about development of the cathode spots of the vacuum arc discharge. It is found that the speed of the cathode spots' motion depends on the cathode temperature.
\end{abstract}

Key words: cathode spot, vacuum arc, integrally cold cathode

\section{INTRODUCTION}

The vacuum arc discharge is a self-maintained discharge developing in the cathode material vapor. The emission center of the discharge is a cathode spot with small dimensions of $10^{-6} \ldots 10^{-3} \mathrm{~m}$. For very short period of time, the temperature in the cathode spot exceeds the temperature of boiling, what results in the intensive evaporation of the cathode material [1] - [3].

The cathode spot includes an emitting zone and an adjacent collisionless layer of the spatial charge, where the cathode drop is localized and the energy is transmitted to the ions. Amount of this energy is enough to heat the cathode to the temperature ensuring energy distribution of the free electrons in the cathode body and reproduction of required amount of the evaporable material.

Discharge combustion is impossible when the temperature in the cathode spot is below certain critical temperature, which is specified by thermophysical properties of the cathode material and electrical parameters of the circuitry: $U_{\text {power supply }}>U_{\mathrm{c}}, I_{\text {power supply }}>I_{\min }$, where $U_{\text {power supply }}$ - supply voltage; $U_{\mathrm{c}}-$ cathode drop; $I_{\text {power supply }}$ - supply current; $I_{\min }$ - the minimum current value required for existence of the discharge. Value of the power released on the cathode is determined by the cathode drop, which value is close to the value of metal ionization potential, and the value of discharge current. Motion of the cathode spots is caused by spontaneous dying out of some cells and formation of others [4].

Exposure of the cathode surface to strong heat source results in the complex physical-chemical processes leading to change of structure and phase 
composition of the surface layer. These changes can have an effect on both speed of cathode spots' motion and processes in the arc discharge plasma. However, changing speed of cathode spots' motion is connected with the magnetic field generated by discharge current.

Also it should be noted, that the vacuum arc discharge with the integrally cold cathode exists on the cathode working surface until its temperature is insufficient to maintain the current flow between the electrodes due to thermal electron emission only. When the certain temperature is reached, this type of the discharge turns into the arc discharge with diffuse connection on the cathode [5]. Therefore, the vacuum arc discharge with the integrally cold cathode should be considered as a transient stage in the development of the arc discharge with the hot cathode, and dynamics of cathode spots' development with time, as the cathode is heated up, should be taken into account.

\section{DESCRIPTION OF THE EXPERIMENTAL FACILITY}

A vacuum arc facility of extended design (Fig. 1) operating in pulse periodic mode and generating the directional belt stream of plasma [6] was used to research the cathode spots. External magnetic field generated by the solenoid 1 initiates longitudinal motion of the cathode spot 6 along the working surface of the extended cylindrical cathode 2 from the igniter electrode 5 towards the current lead-in 3 to the arc-extinguishing screen 4 . The interval between igniting impulses depends on the lifetime of the cathode spots on the working cathode surface.

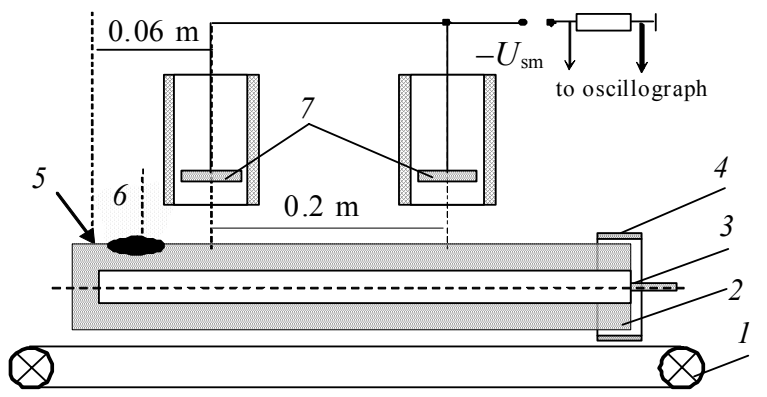

Fig.1 - The vacuum arc plasma sources of extended design: 1 - magnetic system; 2 cathode; 3 - current lead-in; 4 - arc- extinguishing screen; 5 workpiece; 5 - igniter electrode; 6 - cathode spot; 7 - probes.

The researches have shown, that the maximum life time $\tau$ of the cathode spots on the working surface of the water-cooled zirconium cathode of length $0.4 \mathrm{~m}$ and diameter $0.06 \mathrm{~m}$ exceeded $100 \mathrm{~ms}$ at the discharge current in $200 \mathrm{~A}$. And observed shortening of current pulses duration was connected with increase of the cathode working temperature.

Taking into account that first current pulses were long, the VHS video camera was used to record motion of the cathode spots along the working sur- 
face. TV standards have interlaced scanning: one second contains 25 frames and 50 fields (half-frames) with even and odd lines. Therefore, a computer monitor displays each frame with two fixed moments of the observed motion: the first moment corresponds to the odd field, and the second moment - to the even field.

Also, motion of the cathode spots was researched with the help of the electrical probes 7 (Fig. 1) located above the cathode from the igniter electrode to the arc-suppressing shield. Design and location of the probes made it possible to record the ion component maximum of the plasma stream on the oscillograph screen, if coincidence of the moving cathode spots and the axis of the probe receiving surface would be provided.

\section{RESEARCH RESULTS}

The researches carried out revealed two types of discharges described by different existence conditions: the arc discharge on dielectric films of contaminations appearing at the first instant of time and the main arc discharge from the cathode material.

When the facility is switched on for the firs time, a discharge with the cathode spots, moving chaotically along the whole surface of the cathode and the screens, appears on the impure cathode surface. This discharge can exist at lower value of the discharge current than the discharge appearing on the cathode cleaned of dielectric films. The value of the discharge current determines the quantity of the spots existing simultaneously on the working cathode surface. On the thin-film coatings, the value of current being closed on each cathode spot can be only of some amperes, and there increasing of the simultaneously existing cathode spots is watched.

Appearance of high-speed and uncontrolled luminous formations on the cathode surface can be connected with sliding discharge. This kind of discharge is formed as a result of partial sedimentation of positive charged particles on the surface of impurities. In this case, double electrical layers with high intensity of the electric field are formed. The breakdown of this layer even if in one point initiates avalanche of these breakdowns. As a result on the working cathode surface in the impure zones the uncontrolled surface high-speed discharges appear. These discharges eliminate dielectric films, but do not cause deep erosion processes on the cathode surface.

After cleaning of the working cathode surface, nature of the cathode spots' motion along the cathode surface and quantity of the spots are changed totally.

During the all subsequent instants of time, the cathode spot always moves to the less heated zone of the cathode, therefore its speed is determined by the value of the power supplied and the time required to heat the cathode cell up to the working temperature of vaporization. 
Time required to heat the surface depends on the initial temperature in the point of the cathode spot. And it should be noted that, when the phase state of metal is changing from solid to liquid, the value of the heat conductivity coefficient is changing too. For the emission center continuously moving along the working surface, appearance of a new spot is caused by a hot spot and some external factors. Here it should be emphasized, that the following processes should be considered in motion of the cathode spot:

- electron emission from the existing spot;

- generation of excessive charged particles in the current channel in plasma from the side of the magnetic field induction maximum;

- heating of a new zone on the cathode surface (the processes connected with dimensional changes of the cathode spot are important here);

- decrease of the power supplied to the old spot and its cooling-down;

- equalizing of the temperature values in the cathode spots;

- moving of the cathode spot to the ready zone.

If for the frame-by-frame consideration we take only those frames where the beginning coincides with the arising current pulse, then the consecutive frames will show the nature of spots' motion [7].

As a result of videotape processing, there was obtained frame scanning that allowed to observe the transformation of the cathode spots in time and to estimate the speed of their moving along the working cathode surface to within $20 \mathrm{~ms}$ - the time of one field mapping. The shooting implemented at the fixed values of the discharge current and the external magnetic field.

The change of nature of the cathode spots' motion along the working surface with increasing of the working temperature of the cathode is shown in Fig. 2. The first pulse of current (pulse duration $\tau=80 \mathrm{~ms}$ ) fixed on the cold cathode corresponds to tree frames of video series (Fig. 2.a).

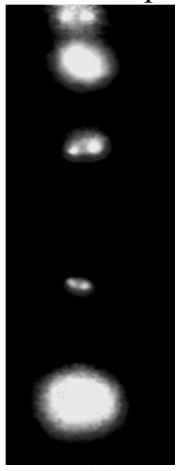

(a)

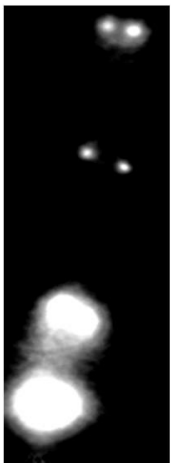

(b)

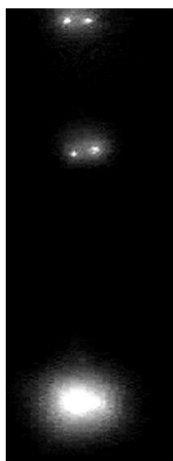

(c)

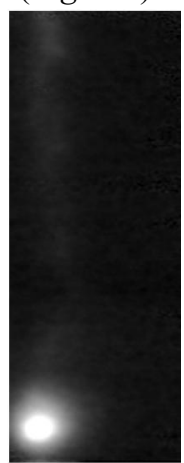

(d)
Fig. 2- Changing of cathode spots moving along the working surface of the extended cathode with changing working temperature of the cathode:a $-\tau=80$ $\mathrm{ms} ; \mathrm{b}-\tau=60 \mathrm{~ms} ; \mathrm{c}-$ $\tau=40 \mathrm{~ms} ; \mathrm{d}-\tau=20$ $\mathrm{ms}$

The group cathode spot that can be watched at the bottom of the frame is connected with its delay near the arc-extinguishing screen. Continuous moving 
of the cathode spots on the limited working cathode surface results in forming some average equilibrium temperature on it. The temperature is determined by the geometrical sizes of the cathode and also by the conditions of its cooling.

As the cathode temperature increases, quantity of moments of cathode spots' motion for one pulse is decreased. Fig. $2 b$ is made of two frames and has three moments of motion ( $\tau=60 \mathrm{~ms}$ ); Fig. $2 c$ includes two moments of motion ( $\tau=40 \mathrm{~ms}$ ); Fig. $2 d$ corresponds to one frame and one moment of motion ( $\tau=$ $20 \mathrm{~ms}$ ). And the afterglow heat time left by the cathode spot is clearly seen here.

Motion of the cathode spots was researched with the help of the probes as well. Design and location of the probes made it possible to record the ion component maximum of the plasma stream on the oscillograph screen, if coincidence of the moving cathode spots and the axis of the electrical probes receiving surface would provided. Fig. 3 shows oscillograms of the current pulses depending on the temperature of the zirconium cathode.

Smooth current rise, observed on all photos at the interval from the igniter electrode to the first probe, is characterized with moving of the cathode spots on the initial zone and connected with warm-up of the cathode. As the cathode temperature rises, noise components of the signal on the oscillograms go down, and the directed velocity of the cathode spots' motion along the working surface towards the current lead-in increases.

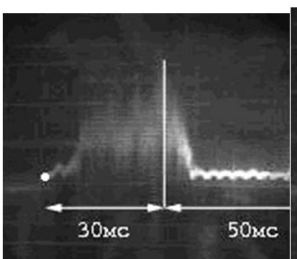

(a)

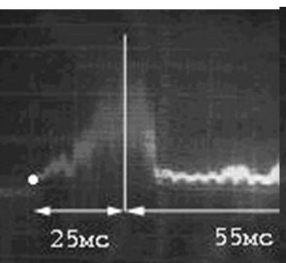

(b)

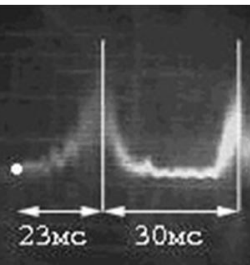

(c)

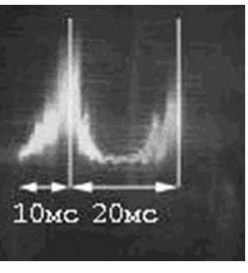

(d)

Fig. 3 - Oscillograms describing the cathode spots' motion along the working surface as the cathode temperature is changing

A cathode spot is displaced towards the maximum of the magnetic field induction $\Sigma \vec{B}_{\max }=\vec{B}_{\mathrm{pl}}+\vec{B}_{\text {cath }}$ [8], generated by the current channel of the plasma stream $I_{\mathrm{pl}}$ and the current flowing in the body of the cathode $I_{\text {cath }}$ (Fig. 4a).

An elementary particle in the plasma stream is affected by the Lorentz force and the electric field force: $\vec{F}_{r}=e\left[\vec{V}_{z} \times \vec{B}\right], \vec{F}_{z}=e\left[\vec{V}_{r} \times \vec{B}\right], \vec{F}_{E}=e \vec{E}$.

To calculate the motion path of the electrons leaving the cathode spot, the following formulas are used: 


$$
\begin{aligned}
& a_{z}=\frac{\vec{F}_{z}}{m_{e}}+\frac{\vec{F}_{\mathrm{E}}}{m_{e}}=\frac{e\left[\vec{V}_{r} \times \vec{B}\right]}{m_{e}}+\frac{e \vec{E}_{E}}{m_{e}}, a_{r}=-\frac{\vec{F}_{r}}{m_{e}}=\frac{e\left[\vec{V}_{z} \times \vec{B}\right]}{m_{e}}, \\
& \vec{V}_{z}=\vec{V}_{0 z}+a_{z} \Delta t, \quad \vec{V}_{r}=\vec{V}_{0 r}+a_{r} \Delta t, \\
& z=z_{0}+\vec{V}_{z} \Delta t, y=y_{0}+\vec{V}_{y} \Delta t,
\end{aligned}
$$

$m_{e}$ is mass of the charged particle (electron); $\vec{F}_{L}$ is projection of the Lorentz force to the axes $z$ and $r ; \vec{V}$ is projection of the charged particle motion velocity to the axes $z$ and $r ; \vec{V}_{0}$ is projection of the charged particle motion velocity to the axes $z$ and $r$ in the previous instant of time; $a$ is acceleration of the charged particle along the axes $z$ and $r ; \Delta t$ is time step; $z$-position of the charged particle on the axis $z ; z_{0}$ is projection of the position of the charged particle on the axis $z$ in the previous instant of time; $y_{0}$ is position of the charged particle on the axis $r ; r_{0}$ is projection of the position of the charged particle on the axis $r$ in the previous instant of time.

Results of the calculations describing existence conditions of the cathode spots on the working surface are shown on the Fig. $4 \mathrm{~b}$ and $4 \mathrm{c}$.

In the process of displacement of the cathode spot, transformation of its dimensions is observed, which changes conditions of the electron emission.

The cathode in the zone of the cathode spots is subject to intensive destruction. Quantity of ions generated in the ionization zone and being the main source of energy in the spot cannot exceed quantity of the evaporated atoms; and dimensional change of the cathode spot leads to change of ionization degree in the plasma stream. In turn, the maximum temperature in the small cathode spots can be reached for shorter time interval than in the bigger spots. In addition, reduction of the cathode spot dimensions and changes of the ion current density influence the electric field intensity. Thus, temperature and dimensions of the cathode spot control atom evaporation and specify conditions of the discharge maintaining.

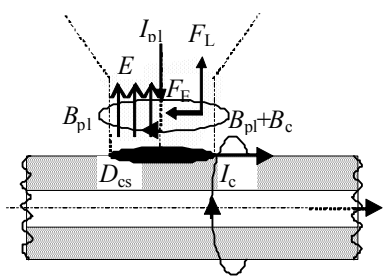

(a)

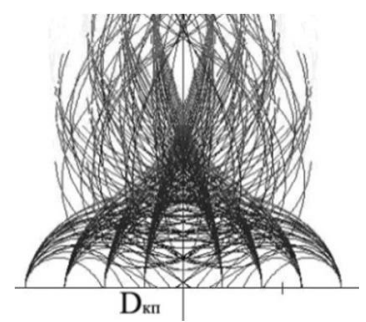

(b)

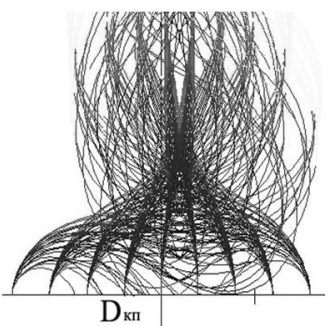

(c)

Fig. 4-Simulation of electron motion from the cathode spot zone: $a$-mathematical model of the calculation with an allowance of the Lorentz force; calculation of the electron path from the cathode without $(b)$ and with $(c)$ an allowance of the current flowing in the cathode 
The power supplied directly to the evaporating surface is spend on heating the cathode in the cathode spot up to the temperature required for reproduction of necessary amount of evaporating material in unit time. Time spent on heating the cathode up to the required temperature is defined by the values of the initial cathode temperature and the power supplied from the discharge. Thus, speed of the cathode spots' motion is defined by the values of the temperature in the spots and the discharge current.

The cathode spot as a heat source of influence on the cathode surface is simulated as a circle of the radius $\mathrm{R}$ (Fig. 5). Within the circle, the value of the heat flow, which is delivered from the discharge and interacting with the surface, is constant for any instant of time $-\lambda(d T / d h)_{h=0}=q,(0 \leq r \leq R)$, and outside the circle it is equal zero. The cathode, with respect to effective size of the heat source, is a semi-infinite body. Therefore, at the infinite distance from the surface, the value of the heat flow is equal zero and the value of the temperature is constant $(d T / d h)_{\mathrm{h}=0}=0,(-\infty \leq r \leq-R$ and $R \leq r \leq \infty)(-R \leq r \leq R)$; $(d T / d h)_{h=\infty}=0 ; T_{h=\infty}=T_{0}$. In the initial state, the cathode surface has the same temperature in all points $T_{t=0}=T_{0}$.

To calculate the temperature field at the action of the heat source the following relation was used:

$T(h, t)=\frac{2 q \sqrt{a t}}{\lambda}\left(\operatorname{ierfc}\left(\mathrm{h} / 2 \sqrt{\mathrm{at})}-\operatorname{ierfc}\left(\sqrt{\mathrm{h}^{2}+R^{2}} / 2 \sqrt{a t}\right)\right), \operatorname{ierfc}(\mathrm{x})=\frac{\exp \left(-\mathrm{x}^{2}\right)}{\sqrt{\pi}}-x \operatorname{erfc}(x)\right.$,

wherehis heating depth; $a=\lambda / \rho c$ is thermal diffusivity; ierfcis theintegrated form of the error Gaussian function.

As the cathode spot moves along the working surface, the hightemperature zones in the form of extended isotherms are left behind the spot (Fig. 6 and Fig. 2d).

To develop a self-consistent mathematical model, that can sufficiently describe the process in the researched vacuum-arc discharge, there should be considered interrelation of the processes in the discharge plasma with the processes defining the temperature in the cathode spot and the average cathode temperature. So far, there are no clear conceptions about such interrelation of these processes. It can be indirectly evidenced by the fact that a lot of types of electron emission from the cathode spot are used to explain the large values of the current density observed in the cathode spot.

In this case, the combined equations including the equation for the electric field intensity at the cathode surface should be used. The electric field intensity depends on spatial distribution of the charged particles and the cathode spot dimensions, as changed in the cathode dimensions leads to changes in intensity, density of emission and ion currents, energy balance in the generation zone, heat balance on the cathode surface, and cathode temperature equations. 


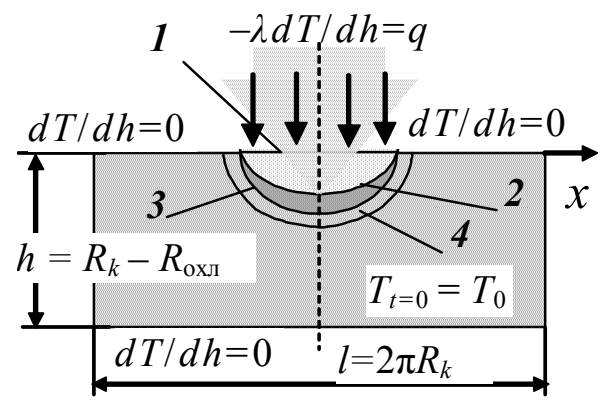

Fig. 5 - Model of the heat flow influence upon the surface: 1 - initial position of the cathode spot on the surface; 2 - lower limit at boiling; 3 lower limit of the liquid phase; 4 - limit of cathode heating up to the emission temperature.

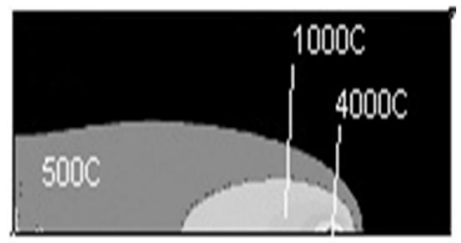

Fig. 6 - Calculated isotherms on the cathode surface, as the steady heat source of the diameter $5 \cdot 10^{-6}$ $\mathrm{m}$ and the speed $10 \mathrm{~m} / \mathrm{sec}$ moves.

In the considered model, only the values of the discharge current $I_{\mathrm{d}}$ and the initial physical constants describing the cathode material can be specified. All other parameters are to be calculated: cathode drop $U_{\mathrm{k}}$, dimensions of the cathode spot $D_{\text {cs }}$, current density $j$, fraction of electron current $I_{\mathrm{e}}$, degree of plasma ionization $\alpha$, electric field intensity at the cathode surface $E$, temperature in the cathode spot $T_{\mathrm{cs}}$ and the cathode itself $T_{\mathrm{k}}$.

The electric field at the solid surface can be formed due to the exterior potential difference as well as due to the field of positive ions located at the cathode surface. Such layer of ions is formed when the field-emission cathode is evaporated in the process of heating by the field-emission current. Subsequent ionization of the evaporated atoms leads to formation of the layer of dense nonequilibrium plasma at the cathode surface. The intense electric field in the border zone induces additional strengthening of the field emission. This process of transition from the regular field emission to higher densities of the emission current results in formation of the vacuum arc in the discharge gap.

Thus, density of the emission current and type of the existing emission are defined by the values of temperature in the cathode spot $T_{\mathrm{cs}}$ and electric field intensity $E$ generated at the cathode surface: $j_{\mathrm{e}}=f\left(T_{\mathrm{cs}}, E\right)$.

In its turn, electric field intensity $E=f\left(U_{\mathrm{k}}, r_{\mathrm{cs}}, j_{\mathrm{i}} / j_{\mathrm{e}}\right)$, where $U_{\mathrm{k}}$ - potential drop in the space-charge zone; $r_{\mathrm{cs}}$-radius of the cathode spot; $j_{\mathrm{i}} / j_{\mathrm{e}}-$ density relation of ion and electron currents, and is defined by the McCown equation.

\section{CONCLUSIONS}

The researches of the vacuum-arc plasma source of exceeded design operating in pulse mode and generating the directed belt stream have allowed to 
reveal the dependence of the development dynamics and the velocities of the cathode spots' motion along the working cathode surface on its temperature and to make the following suppositions:

1. The arc discharge arisen at the initial moment on the uncleaned cathode surface is to be considered as the self-maintained arc discharge.

2. After cleaning of the working surface, the main arc discharge is developed on the cathode material. Motion of the cathode spots is getting "ordered", and the prevalent motion of the spots towards the current lead-in becomes apparent.

3. Time of development of the main arc discharge from the cathode material and speed of the cathode spots' motion along the working cathode surface depends on the cathode temperature.

4. The presence of transient processes, while the arc discharge becomes stable, leads to irregular erosion of material from the cathode surface and therefore to irregular deposited coating on the product being processed.

\section{REFERENCES}

[1] V.L. Granovsky, Electric current in gas, Moscow, 1971, P. 99-141.

[2] J. Cobain Introduction in physics of vacuum arc // Vacuum arcs. Moscow, Mir, 1982. P.19-39.

[3] G. Ecker, Vacuum Arc Theory// Vacuum arcs. Moscow, Mir, 1982. P.269-384.

[4] G.A. MesjazEctons. Ekaterinburg: UIF Nauka, 1993, Part 2, 244 p.

[5] A.M. Dorodnov, A.I. Kuznezov,Appl. Phys. Lett., 1979, Vol. 5. No16, P.1001-1006.

[6] N.Z. Vetrov, A.A. Lisenkov.et al., Patent of the Russian Federation No. 2180472 of 07.02.2000, MKI H05H1/50, Vacuum-arc source of plasmas. Published in 2002. B.I. No7.

[7] A.A. Lisenkov, Vacuum technology, 2004, Vol. 14,No4, P. 221-226.

[8] G.L. Saksagansky,Electrophysical vacuum pumps. Moscow, Energoatomizdat, 1988. $277 \mathrm{p}$. 